\title{
PARÂMETROS DE DESEMPENHO EM MÉTODO UHPLC-UV PARA QUANTIFICAÇÃO DEAMINOÁCIDOS LIVRES E AMINAS BIOATIVAS EM QUEIJOS MUSSARELA, PRATO, PARMESÃO E GORGONZOLA
}

\section{Performance parameters of a UHPLC-UV method for quantification of free amino acids and bioactive amines in Mozzarella, Prato, Parmesan and Gorgonzola cheeses}

\begin{abstract}
Gisela de Magalhães Machado Moreira ${ }^{1 *}$, Denise Sobral ${ }^{1}$, Renata Golin Bueno Costa , Junio César Jacinto de Paula ${ }^{1}$, Christian Fernandes ${ }^{2}$, Maria Beatriz Abreu Glória ${ }^{2}$
\end{abstract}

\section{RESUMO}

Foram apresentados parâmetros de desempenho para um método de cromatografia líquida de ultra eficiência para a determinação de 19 aminoácidos, 10 aminas bioativas e o íon amônio nos queijos Parmesão, Gorgonzola, Prato e Mussarela. As curvas analíticas apresentaram coeficiente de correlação $\geq 0,97$. O método foi seletivo para todos os analitos nos quatro tipos de queijo. Os coeficientes de variação (repetitividade) encontraram-se todos abaixo de 10\%. A recuperação dos compostos foi superior a $70 \%$ em mais de $76 \%$ das determinações. O queijo que apresentou melhores índices de recuperação foi o Prato. Os menores limites de detecção e quantificação encontrados foram para a glutamina, 0,07 e $0,22 \mathrm{mg} / 100 \mathrm{~g}$ respectivamente, enquanto os maiores foram para o ácido aspártico, 0,81 e 2,46 mg/100 g. Desta forma o método é adequado para determinação dessas substâncias nos queijos estudados.

Palavras-chave: proteólise; validação; efeito matriz; queijo brasileiro.

1 Empresa de Pesquisa Agropecuária de Minas Gerais, Instituto de Laticínios Cândido Tostes (EPAMIG/ILCT), Rua Tenente Luiz de Freitas, 116, 36045-560, Juiz de Fora, MG, Brasil. E-mail: giselammachado@hotmail.com

2 Universidade Federal de Minas Gerais, Faculdade de Farmácia, Laboratório de Bioquímica de Alimentos, Belo Horizonte, MG, Brasil.

* Autor para correspondência. 


\begin{abstract}
Performance parameters were presented for anultra high-performance liquid chromatographic method for determination of 19 amino acids, 10 bioactive amines and ammonium ion in Parmesan, Gorgonzola, Prato and Mozzarella cheeses. Analytical curves showed correlation coefficient $\geq 0.97$. The method was selective for all analytes in the four types of cheese. The coefficients of variation in a repeatability experiment were all below $10 \%$. The recovery of the compounds was over $70 \%$ in more than $76 \%$ of the determinations. The cheese with best recovery rates was Prato. The lowest limits of detection and quantification were found for glutamine, 0.07 and $0.22 \mathrm{mg} / 100 \mathrm{~g}$ respectively, whereas the highest limits were for aspartic acid, 0.81 and $2.46 \mathrm{mg} / 100 \mathrm{~g}$. Based on these results, the method is suitable for determination of these substances in the cheeses studied.
\end{abstract}

Keywords: proteolysis; validation; matrix effect; Brazilian cheese.

\section{INTRODUÇÃO}

A proteólise é o evento mais complexo que ocorre durante a maturação de queijos e se dá pela ação de enzimas proteolíticas advindas de fontes diversas: do próprio leite, do coagulante, adicionadas e/ou de culturas starter, secundárias ou de contaminantes (McSWEENEY, 2011). Os aminoácidos liberados pela proteólise em queijos são de especial relevância tanto para a formação de flavour quanto do ponto de vista toxicológico, visto que são precursores de aminas biogênicas (VALE; GLÓRIA, 1998; YVON; RIJNEN, 2001; ROIG-SAGUÉS et al., 2002; LINARES et al., 2012).

Aminas biogênicas são derivadas da descarboxilação de aminoácidos pela ação de enzimas que estão presentes em culturas iniciadoras ou contaminantes (ALVAREZ; MORENO-ARRIBAS, 2014). São relevantes do ponto de vista sanitário e toxicológico, podendo indicar a qualidade da matéria prima e as condições higiênico-sanitárias prevalentes na produção de alimentos. Algumas aminas, em concentrações elevadas, podem causar efeitos adversos à saúde humana, como a histamina e a tiramina. Estas são causadoras de sintomas tais como febre, dores de cabeça, alterações na pressão arterial, vômitos, náu- seas e diarreia, por suas ações vasoativas ou neuroativas (GLÓRIA, 2006; EFSA, 2011).

Os queijos possuem ambiente favorável para a formação das aminas devido à presença de aminoácidos livres, bactérias capazes de promover a descarboxilação destes e outros fatores como $\mathrm{pH}$, concentração salina, atividade de água, temperatura e tempo de estocagem/maturação, densidade bacteriana e a presença do cofator piridoxal fosfato (VALE; GLÓRIA, 1998; ROIG-SAGUÉS et al., 2002; LINARES et al., 2012; LOIZZO et al., 2013).

A determinação de aminoácidos livres em queijos é importante por embasar avaliações a respeito da profundidade da proteólise durante a maturação, e também por poder ser usada como medida da qualidade nutricional, por apontar possíveis adulterações ou transformações ocorridas durante a fabricação e a maturação, e por prever o potencial de formação de aminas biogênicas e compostos de aroma (YVON; RIJNEN, 2001; KABELOVÁ et al., 2009). Além disto, os aminoácidos livres por si só contribuem para o aroma de queijos; e o seu catabolismo é o principal processo de formação de flavour durante a maturação. $\mathrm{O}$ catabolismo de aminoácidos ocorre por duas rotas bioquímicas, sendo a principal a de 
transaminação, que é a reação preferencial de degradação de aminoácidos por bactérias lácticas, originando $\alpha$-ceto ácidos que serão posteriormente degradados a vários compostos responsáveis pelo aroma em queijos (YVON; RIJNEN, 2001).

Métodos cromatográficos são os mais utilizados para a determinação de aminas em diversas matrizes alimentares (CUSTÓDIO et al., 2007; LOIZZO et al., 2013). Nos últimos anos, a cromatografia também tem sido comumente empregada para a determinação simultânea de aminas e aminoácidos livres (JIA et al., 2011; FIECHTER et al., 2013; HE et al., 2016). A determinação simultânea dessas duas classes de compostos é desafiadora devido à diversidade estrutural, sendo comum ocorrerem problemas relacionados à separação adequada e à obtenção de tempos de retenção satisfatórios, principalmente em análise de alimentos e matrizes biológicas complexas (MAZZUCCO et al., 2010; TUBEROSO et al., 2015; HE et al., 2016). A derivação química é uma forma de minimizar estes problemas, uma vez que a adição de um grupo funcional às moléculas-alvo pode tornar o método mais sensível e seletivo, diminuindo a interferência endógena (HE et al., 2016). Para amostras complexas como produtos fermentados, onde a coexistência de aminas e aminoácidos pode ser prevista, espera-se que ambos sejam extraídos simultaneamente na etapa de preparo de amostras e a derivação ocorra de forma simultânea para as duas classes de compostos aminados, dependendo do agente derivante empregado (FIECHTER et al., 2013).

Cada tipo de queijo tem seu próprio perfil de aminas biogênicas e aminoácidos livres, resultantes das suas reações específicas de degradação, interconversão e síntese (BAKKER; LAW, 1994). Portanto, torna-se fundamental conhecer essas substâncias e estudar sua presença em queijos de diversos tipos. Nesse contexto, esse trabalho teve como objetivo determinar parâmetros de desempenho para um método de cromatografia líquida de ultra eficiência de determinação simultânea de 19 aminoácidos livres, 10 aminas bioativas e do íon amônio, em quatro tipos de queijos produzidos no Brasil: Mussarela, Prato, Parmesão e Gorgonzola.

\section{MATERIAL E MÉTODOS}

\section{Material}

\section{Reagentes}

Os reagentes utilizados nas análises cromatográficas eram de grau analítico, exceto a acetonitrila, que era de grau cromatográfico. As soluções foram preparadas com água ultrapura obtida de Sistema Milli-Q Plus (Millipore Corp., Milford, MA, EUA). As fases móveis foram filtradas em membrana de $0,22 \mu \mathrm{m}$ de tamanho do poro e $47 \mathrm{~mm}$ de diâmetro (Millipore Corp., Milford, MA, EUA). Os padrões de aminas bioativas e aminoácidos utilizados foram: alanina (98\%), monohidrocloreto de arginina (98\%), ácido aspártico (98\%), ácido glutâmico (99\%), cistina cristalina (98\%), fenilalanina (98\%), glicina (99\%), monohidrocloreto de histidina monohidratado (98\%), isoleucina (98\%), monohidrocloreto de lisina (98\%), leucina $(98 \%)$, metionina $(98 \%)$, prolina $(99 \%)$, serina (99\%), tirosina (98\%), treonina (98\%), valina (98\%), cloridrato de beta-feniletilamina (98\%), serotonina creatinina sulfato mono hidratado (100\%), cloridrato de tiramina $(98 \%)$, dicloridrato de cadaverina (98\%), dicloridrato de histamina (99\%), dicloridrato de putrescina (98\%), sulfato de agmatina (97\%), tetracloridrato de espermina (100\%), tricloridrato de espermidina (98\%) e triptamina (98\%), glutamina (99\%), asparagina (98\%), norvalina $(99 \%)$, todos adquiridos da Sigma Chemical Co. (St. Louis, MO, EUA). Cloreto de amônio (100\%) adquirido da Synth (Diadema, SP, Brasil).A derivação foi feita utilizando kit Waters AccQ.Fluor ${ }^{\circledR}$ contendo 
tampão borato e o derivante 6-aminoquinolilN-hidroxi succinimidilcarbamato (AQC).

\section{Queijos}

Os queijos Mussarela, Prato, Parmesão e Gorgonzola foram produzidos em três repetições no Núcleo Industrial da EPAMIG - Instituto de Laticínios Cândido Tostes, em Juiz de Fora, MG, conforme metodologia descrita em Dutra; Munck (2002).

\section{Métodos}

\section{Preparo de amostras e derivação}

A extração das aminas e aminoácidos nas amostras de queijos ocorreu conforme descrito em Custódio et al. (2007), usando ácido clorídrico $1 \mathrm{~mol} / \mathrm{L}$ como reagente extrator, seguido de agitação em agitador orbital Tecnal ${ }^{\circledR}$ modelo TE-140 (250 rpm por 10 minutos), centrifugação (11.180 x $g$ por 21 minutos a $4{ }^{\circ} \mathrm{C}$ ) e filtragem em papel de filtro qualitativo. Foram realizadas três extrações sucessivas vertendo no mesmo balão volumétrico, sendo adicionado o padrão in- terno L-norvalina para concentração final in column de 25 pmol antes de se completar o volume do balão.

A derivação das aminas e aminoácidos nos extratos ocorreu segundo metodologia descrita em Fiechter et al. (2013). Após neutralização dos extratos com hidróxido de sódio $1 \mathrm{~mol} / \mathrm{L}$ e centrifugação (16.000 $\mathrm{x} g$, por 10 minutos a $4{ }^{\circ} \mathrm{C}$ ), as aminas e aminoácidos foram derivados (derivação pré-coluna) por reação com 6 aminoquinolil$\mathrm{N}$-hidroxisuccinimidilcarbamato (AQC) utilizando o kit Waters AccQ.Fluor ${ }^{\circledR}$. A $5 \mu \mathrm{L}$ de extrato neutralizado foram adicionados $35 \mu \mathrm{L}$ de tampão borato AccQ.Fluor ${ }^{\circledR}$ e $10 \mu \mathrm{L}$ de reagente AQC. Após 1 minuto de descanso, o extrato foi aquecido a $55^{\circ} \mathrm{C}$ por 10 minutos em banho-maria para completar a reação de derivação. As amostras derivadas foram filtradas em filtros de seringa com $0,22 \mu \mathrm{m}$ de poro (Whatman ${ }^{\circledR}$, GE Healthcare, Reino Unido) para vial Total Recovery ${ }^{\circledR}$ Waters e analisadas por cromatografia líquida de ultra eficiência.

Tabela 1 - Gradiente de concentração das soluções de fases móveis empregadas na separação das aminas bioativas e aminoácidos livres nos extratos de queijo

\begin{tabular}{ccc}
\hline Tempo (minutos) & \% A (tampão acetato $\mathrm{pH} \mathrm{4,8)}$ & \% B (acetonitrila) \\
\hline inicial & 100 & 0 \\
2,5 & 100 & 0 \\
4 & 97 & 3 \\
9 & 70 & 30 \\
10 & 70 & 30 \\
10,5 & 0 & 100 \\
11 & 0 & 100 \\
11,5 & 100 & 0 \\
\hline
\end{tabular}

Reequilíbrio às condições iniciais por mais $1,5 \mathrm{~min}$.

Tempo total de corrida: 13 minutos.

Fonte: adaptado de Fiechter et al. (2013). 


\section{Análise cromatográfica}

A análise cromatográfica das aminas e aminoácidos derivados foi realizada conforme descrito em Fiechter et al. (2013). Foi utilizado cromatógrafo líquido de ultra eficiência Waters Acquity ${ }^{\circledR}$ Ultra Performance LC (UPLC ${ }^{\circledR}$ ) equipado com um detector Acquity ${ }^{\circledR}$ ultravioleta ajustável (TUV) (Waters, Milford, MA, EUA). Para a separação utilizou-se coluna de fase reversa Acquity UPLC ${ }^{\circledR}$ BEH C18 (2,1 x 50 mm, 1,7 $\mu \mathrm{m})$. A fase móvel foi constituída de: A) tampão acetato de sódio $0,1 \mathrm{~mol} / \mathrm{L}$ em água ultrapura com $\mathrm{pH}$ ajustado para 4,8 com ácido acético p.a. e B) acetonitrila de grau cromatográfico, ambos filtrados à vácuo em membrana com poro de 0,22 $\mu \mathrm{m}\left(\mathrm{GV}\right.$ Durapore Merck $^{\circledR}$ ) e sonicados por 30 minutos. Foi empregado modo de eluição gradiente, conforme apresentado na Tabela 1. O volume de injeção de amostra foi $2 \mu \mathrm{L}$, a vazão da fase móvel foi de $1 \mathrm{~mL} / \mathrm{min}$ e o comprimento de onda de detecção foi $249 \mathrm{~nm}$ a uma taxa de amostragem de 40 pontos/segundo. O software Waters Empower 2 foi utilizado para controle do UHPLC e aquisição dos dados.

A identificação das aminas e aminoácidos foi feita por comparação do tempo de retenção dos picos dos analitos na amostra em relação aos da solução padrão e também pela adição da substância suspeita à amostra. O cálculo da concentração das aminas e aminoácidos foi feito por interpolação nas respectivas curvas analíticas.

\section{Desempenho do método}

Os experimentos de avaliação do desempenho do método analítico foram conduzidos segundo orientações do Manual de Garantia da Qualidade Analítica (BRASIL, 2011). Os dados obtidos foram tratados no software estatístico Minitab ${ }^{\circledR}$ versão 14 (State College, PA, EUA).

\section{RESULTADOS E DISCUSSÃO}

\section{Linearidade, Sensibilidade e Faixa de Trabalho}

Os quatro tipos de queijos estudados foram analisados quanto ao teor de aminoácidos livres e aminas bioativas, no sentido de determinar o queijo com menor concentração de analitos para ser utilizado na determinação da curva padrão na matriz, devido à impossibilidade de se obter uma matriz branca. As análises foram realizadas após 15, 8 e 29 dias de fabricação dos queijos Mussarela, Prato, Parmesão, respectivamente e 14 dias após perfuração do queijo Gorgonzola. $\mathrm{O}$ queijo Mussarela foi o que apresentou os menores teores de todos os analitos (menor que 2 pmol in column, ponto mínimo da curva de calibração). Dessa forma esta matriz foi escolhida para determinação da curva de calibração em matriz.

Para construção da curva de calibração foram escolhidos 6 níveis de concentração (FIECHTER et al., 2013) com concentração final in column de 2, 20, 40, 60, 80 e 100 pmol. Norvalina foi usada como padrão interno em concentração constante de $25 \mathrm{pmol}$ in column. Considerando a técnica utilizada, as diluições aplicadas e a massa molar de cada analito, os pontos mínimo e máximo da curva em $\mathrm{mg}$ analito/100 g queijo estão apresentadas na Tabela 2. Cada solução foi preparada 3 vezes e injetada 2 vezes aleatoriamente. Os dados obtidos foram tratados pelo Método dos Mínimos Quadrados Ponderados (BRASIL, 2011) sendo que para todos os analitos as equações lineares foram significativas. Os parâmetros das equações das retas de calibração - o intercepto e a inclinação (sensibilidade), bem como o coeficiente de determinação obtido para cada analito, estão também descritos na Tabela 2 . 
Tabela 2 - Mínimo e máximo da faixa de trabalho, intercepto (a) e inclinação (b) da curva analítica e coeficiente de determinação $\left(\mathrm{R}^{2}\right)$ para cada analito calculado em extrato de matriz fortificado

\begin{tabular}{|c|c|c|c|c|c|}
\hline \multirow{2}{*}{ Analitos } & \multicolumn{2}{|c|}{ Faixa trabalho $(\mathrm{mg} / 100 \mathrm{~g})$} & \multicolumn{3}{|c|}{ Curva analítica } \\
\hline & mínimo & máximo & $\mathbf{a}$ & b & $\mathbf{R}^{2}$ \\
\hline Íon Amônio & 0,3 & 17,0 & 0,134 & 0,023 & 0,992 \\
\hline Aminoácidos & mínimo & máximo & $\mathbf{a}$ & b & $\mathbf{R}^{2}$ \\
\hline Ácido Aspártico & 2,7 & 133,1 & 0,072 & 0,025 & 0,997 \\
\hline Ácido Glutâmico & 2,9 & 147,1 & 0,044 & 0036 & 0,998 \\
\hline Alanina & 1,8 & 89,1 & 0,079 & 0,034 & 1,000 \\
\hline Arginina & 3,5 & 174,2 & 0,057 & 0,035 & 0,998 \\
\hline Asparagina & 2,6 & 132,1 & $-0,058$ & 0,034 & 0,996 \\
\hline Cistina & 4,8 & 240,3 & 0,007 & 0,065 & 0,998 \\
\hline Fenilalanina & 3,3 & 165,2 & 0,071 & 0,043 & 0,992 \\
\hline Glicina & 1,5 & 75,1 & 0,096 & 0,019 & 0,999 \\
\hline Glutamina & 2,9 & 146,1 & 0,285 & 0,043 & 0,984 \\
\hline Histidina & 3,1 & 155,2 & 0,024 & 0,037 & 0,996 \\
\hline Isoleucina & 2,6 & 131,2 & 0,027 & 0,040 & 1,000 \\
\hline Leucina & 2,6 & 131,2 & 0,091 & 0,048 & 0,995 \\
\hline Lisina & 2,9 & 146,2 & 0,203 & 0,057 & 0,996 \\
\hline Metionina & 3,0 & 149,2 & 0,018 & 0,039 & 0,999 \\
\hline Prolina & 2,3 & 115,1 & 0,007 & 0,038 & 0,999 \\
\hline Serina & 2,1 & 105,1 & 0,005 & 0,030 & 1,000 \\
\hline Tirosina & 3,6 & 181,2 & 0,030 & 0,038 & 0,993 \\
\hline Treonina & 2,4 & 119,1 & 0,039 & 0,034 & 1,000 \\
\hline Valina & 2,3 & 117,2 & 0,082 & 0,034 & 1,000 \\
\hline Aminas & mínimo & máximo & a & b & $\mathbf{R}^{2}$ \\
\hline Agmatina & 2,6 & 130,2 & $-0,007$ & 0,035 & 1,000 \\
\hline Cadaverina & 2,0 & 102,2 & 0,021 & 0,063 & 0,993 \\
\hline Espermidina & 2,9 & 145,2 & $-0,039$ & 0,065 & 0,971 \\
\hline Espermina & 4,0 & 202,3 & $-0,066$ & 0,056 & 0,998 \\
\hline Feniletilamina & 2,4 & 121,2 & 0,002 & 0,040 & 0,996 \\
\hline Histamina & 2,2 & 111,2 & $-0,003$ & 0,030 & 0,999 \\
\hline Putrescina & 1,8 & 88,2 & 0,013 & 0,067 & 0,997 \\
\hline Serotonina & 3,5 & 176,2 & $-0,014$ & 0,036 & 0,993 \\
\hline Tiramina & 2,7 & 137,2 & 0,046 & 0,041 & 0,996 \\
\hline Triptamina & 3,2 & 160,2 & $-0,031$ & 0,049 & 0,997 \\
\hline
\end{tabular}




\section{Seletividade e efeito matriz}

De acordo com o Manual de Garantia da Qualidade Analítica (BRASIL, 2011), a verificação da seletividade do procedimento analítico deve ser realizada por comparação entre a resposta analítica do extrato da matriz, da matriz fortificada e do analito puro em solvente. O método foi seletivo para todos os analitos estudados em todos os quatro tipos de queijo avaliados, não apresentando deslocamento de pico na matriz em relação ao solvente. Esses resultados foram comprovados por fortificação individual de cada analito em extrato de cada queijo, e um exemplo de cromatograma comparativo entre uma amostra de queijo Parmesão e o pool de aminoácidos e aminas em solução está demonstrado na Figura 1.

Apesar de seletivo, o método apresentou efeito matriz por alteração de intensidade de resposta analítica quando quantificado em extrato de queijo em comparação ao pool em solvente. Foram elaboradas amostras com 3 níveis de fortificação em extrato de cada queijo e em solvente, em 6 repetições. Para o Gorgonzola, a glicina e a prolina foram os únicos analitos entre os 30 analisados que não apresentaram efeito matriz em todos os níveis de fortificação avaliados, enquanto todos os outros apresentaram efeito matriz em pelo menos um nível. Não apresentaram efeito

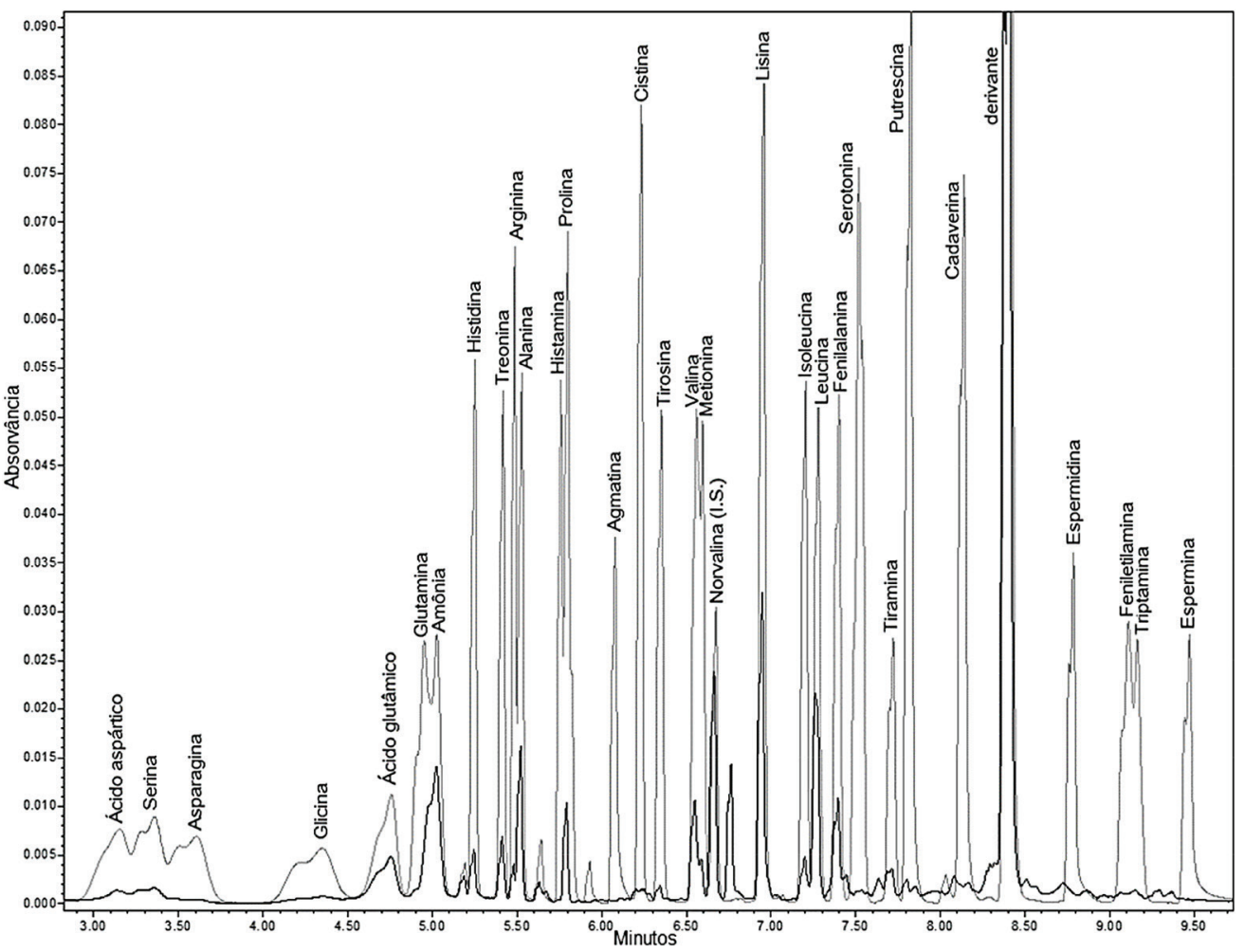

Figura 1 - Cromatograma comparativo de uma amostra de queijo Parmesão após 3 meses de fabricação (picos menores e mais escuros) e um pool dos aminoácidos e aminas em solução (picos maiores em sobreposição, mais claros) 
Tabela 3 - Coeficientes de variação para as determinações em condições de repetitividade de aminas, aminoácidos e íon amônio em 3 níveis de concentração para os queijos Gorgonzola, Mussarela, Parmesão e Prato

\begin{tabular}{|c|c|c|c|c|c|c|c|c|c|c|c|c|}
\hline \multirow{3}{*}{ Analitos } & \multicolumn{12}{|c|}{ Níveis de concentração por tipo de queijo } \\
\hline & \multicolumn{3}{|c|}{ Gorgonzola } & \multicolumn{3}{|c|}{ Mussarela } & \multicolumn{3}{|c|}{ Parmesão } & \multicolumn{3}{|c|}{ Prato } \\
\hline & 1 & 2 & 3 & 1 & 2 & 3 & 1 & 2 & 3 & 1 & 2 & 3 \\
\hline Íon Amônio & 8,18 & 8,81 & 6,08 & 6,35 & 3,49 & 3,10 & 3,80 & 3,98 & 5,07 & 7,37 & 8,89 & 4,79 \\
\hline Aminoácidos & 1 & 2 & 3 & 1 & 2 & 3 & 1 & 2 & 3 & 1 & 2 & 3 \\
\hline Ácido Aspártico & 5,07 & 1,52 & 4,47 & 9,17 & 3,46 & 4,73 & 1,83 & 2,69 & 3,28 & 3,21 & 8,49 & 6,25 \\
\hline Ácido Glutâmico & 4,96 & 7,53 & 4,83 & 6,21 & 4,95 & 2,81 & 4,99 & 3,80 & 3,38 & 8,08 & 6,21 & 4,19 \\
\hline Alanina & 4,43 & 5,95 & 2,82 & 6,35 & 3,66 & 1,85 & 2,07 & 2,66 & 1,67 & 2,59 & 2,15 & 3,57 \\
\hline Arginina & 1,39 & 1,84 & 3,37 & 8,20 & 2,56 & 1,83 & 1,48 & 2,19 & 4,76 & 2,97 & 2,53 & 3,25 \\
\hline Asparagina & 4,34 & 5,39 & 2,69 & 6,73 & 5,97 & 4,00 & 2,65 & 3,55 & 2,90 & 4,33 & 5,22 & 5,47 \\
\hline Cistina & 1,33 & 2,24 & 3,34 & 8,47 & 1,56 & 1,64 & 1,18 & 2,11 & 5,56 & 2,99 & 2,44 & 3,27 \\
\hline Fenilalanina & 3,27 & 3,18 & 2,97 & 8,18 & 2,43 & 1,88 & 1,34 & 1,93 & 6,34 & 2,61 & 2,36 & 3,64 \\
\hline Glicina & 7,89 & 9,85 & 4,01 & 9,86 & 2,90 & 2,79 & 2,58 & 3,56 & 4,28 & 6,83 & 6,55 & 6,09 \\
\hline Glutamina & 9,32 & 6,29 & 3,28 & 7,09 & 2,67 & 3,83 & - & - & - & 7,93 & 9,78 & 4,99 \\
\hline Histidina & 1,82 & 2,88 & 3,40 & 7,93 & 4,63 & 2,92 & 1,18 & 1,88 & 8,74 & 2,97 & 5,21 & 2,77 \\
\hline Isoleucina & 3,28 & 3,68 & 3,58 & 4,97 & 2,30 & 2,83 & 1,26 & 1,81 & 5,90 & 2,44 & 2,40 & 3,91 \\
\hline Leucina & 2,32 & 2,75 & 2,64 & 8,41 & 2,02 & 2,08 & 1,31 & 1,90 & 3,19 & 2,52 & 2,22 & 3,29 \\
\hline Lisina & 0,93 & 3,04 & 4,02 & 9,56 & 4,75 & 2,87 & 1,38 & 2,47 & 3,45 & 2,38 & 2,31 & 5,42 \\
\hline Metionina & 4,09 & 3,84 & 3,14 & 1,23 & 1,51 & 3,40 & 2,84 & 1,92 & 3,82 & 6,39 & 4,42 & 1,99 \\
\hline Prolina & 3,57 & 3,90 & 3,84 & 7,85 & 1,82 & 1,58 & 1,66 & 2,04 & 3,36 & 2,06 & 1,89 & 3,09 \\
\hline Serina & 4,07 & 3,41 & 3,17 & 5,60 & 3,13 & 4,69 & 3,71 & 2,55 & 4,89 & 4,43 & 7,07 & 8,50 \\
\hline Tirosina & 1,67 & 2,34 & 3,42 & 7,92 & 1,50 & 2,29 & 1,28 & 1,88 & 5,91 & 3,67 & 2,34 & 3,10 \\
\hline Treonina & 1,72 & 3,30 & 3,21 & 7,87 & 2,66 & 2,05 & 1,52 & 2,43 & 2,74 & 2,97 & 3,02 & 2,79 \\
\hline Valina & 4,12 & 0,91 & 3,28 & 2,85 & 1,28 & 8,45 & 3,10 & 2,13 & 3,23 & 4,41 & 2,49 & 4,81 \\
\hline Aminas & 1 & 2 & 3 & 1 & 2 & 3 & 1 & 2 & 3 & 1 & 2 & 3 \\
\hline Agmatina & 7,25 & 7,02 & 5,98 & 9,78 & 5,05 & 4,34 & 1,16 & 2,80 & 3,76 & 1,20 & 4,19 & 6,67 \\
\hline Cadaverina & 9,84 & 9,29 & 0,31 & 3,12 & 0,85 & 7,01 & 2,29 & 2,46 & 1,49 & 5,80 & 9,31 & 2,03 \\
\hline Espermidina & 1,17 & 8,75 & 10,26 & 0,28 & 0,69 & 5,69 & 5,12 & 3,69 & 9,44 & 4,20 & 5,63 & 7,30 \\
\hline Espermina & 2,30 & 0,91 & 7,72 & 7,40 & 8,83 & 6,76 & 3,58 & 9,59 & 6,50 & 4,01 & 0,36 & 2,59 \\
\hline Feniletilamina & 6,99 & 6,70 & 1,64 & 6,81 & 8,04 & 6,78 & 3,23 & 2,06 & 6,53 & 9,72 & 9,88 & 2,56 \\
\hline Histamina & 3,84 & 4,58 & 2,01 & 8,20 & 3,53 & 1,51 & 1,19 & 2,28 & 5,52 & 3,56 & 3,03 & 3,40 \\
\hline Putrescina & 5,22 & 5,53 & 3,60 & 1,73 & 5,93 & 7,43 & 2,95 & 1,97 & 1,05 & 2,33 & 6,53 & 1,56 \\
\hline Serotonina & 2,91 & 8,80 & 2,42 & 8,25 & 8,84 & 4,71 & 2,47 & 2,44 & 8,44 & 1,10 & 7,42 & 1,41 \\
\hline Tiramina & 3,99 & 6,98 & 7,94 & 8,50 & 6,32 & 6,32 & 2,04 & 1,77 & 6,03 & 2,38 & 4,70 & 1,67 \\
\hline Triptamina & 2,10 & 3,65 & 5,82 & 8,90 & 0,54 & 8,31 & 1,82 & 2,52 & 6,35 & 4,92 & 9,73 & 2,43 \\
\hline
\end{tabular}

Legenda: (-) não determinado. 
matriz em nenhum nível para a Mussarela: histidina, treonina, isoleucina e tiramina; para o queijo Prato: treonina isoleucina, triptamina e para o Parmesão: histidina, agmatina, fenilalanina e espermina.

\section{Precisão}

A precisão de um método analítico está relacionada com a dispersão dos resultados de ensaios independentes de uma mesma

Tabela 4 - Médias das porcentagens de recuperação e desvio padrão para aminas, aminoácidos e íon amônio em para os queijos Gorgonzola, Mussarela, Parmesão e Prato

\begin{tabular}{|c|c|c|c|c|}
\hline Analitos & Gorgonzola & Mussarela & Parmesão & Prato \\
\hline Í́on Amônio & $71,1 \pm 8,5$ & $68,0 \pm 5,2$ & $54,6 \pm 4,0$ & $79,7 \pm 11,5$ \\
\hline Aminoácidos & Gorgonzola & Mussarela & Parmesão & Prato \\
\hline Ácido Aspártico & $98,8 \pm 2,0$ & $95,4 \pm 3,1$ & $81,6 \pm 2,8$ & $100,5 \pm 9,6$ \\
\hline Ácido Glutâmico & $70,3 \pm 7,9$ & $75,0 \pm 0,8$ & $70,0 \pm 1,6$ & $85,8 \pm 2,7$ \\
\hline Alanina & $71,0 \pm 8,7$ & $71,3 \pm 2,5$ & $65,0 \pm 3,8$ & $72,7 \pm 6,7$ \\
\hline Arginina & $64,2 \pm 8,6$ & $64,4 \pm 5,2$ & $63,6 \pm 5,8$ & $70,3 \pm 4,0$ \\
\hline Cistina & $71,1 \pm 4,9$ & $73,0 \pm 1,1$ & $70,0 \pm 4,1$ & $75,8 \pm 3,7$ \\
\hline Fenilalanina & $74,7 \pm 4,4$ & $70,8 \pm 2,4$ & $70,6 \pm 5,6$ & $74,0 \pm 5,8$ \\
\hline Glicina & $65,5 \pm 7,4$ & $70,8 \pm 2,6$ & $61,5 \pm 4,6$ & $74,4 \pm 5,7$ \\
\hline Histidina & $43,1 \pm 2,2$ & $54,7 \pm 4,2$ & $53,8 \pm 4,6$ & $80,4 \pm 11,1$ \\
\hline Isoleucina & $63,3 \pm 3,7$ & $72,3 \pm 4,4$ & $70,9 \pm 4,8$ & $75,2 \pm 3,2$ \\
\hline Leucina & $73,8 \pm 5,2$ & $70,3 \pm 2,1$ & $70,6 \pm 5,8$ & $70,2 \pm 2,7$ \\
\hline Lisina & $63,8 \pm 7,9$ & $64,6 \pm 5,1$ & $51,7 \pm 3,3$ & $74,2 \pm 9,7$ \\
\hline Metionina & $80,6 \pm 4,6$ & $77,7 \pm 7,0$ & $82,9 \pm 0,6$ & $81,6 \pm 8,4$ \\
\hline Prolina & $60,0 \pm 8,7$ & $71,8 \pm 2,8$ & $61,7 \pm 4,2$ & $79,1 \pm 4,7$ \\
\hline Serina & $70,3 \pm 6,9$ & $73,2 \pm 2,2$ & $72,8 \pm 2,0$ & $80,7 \pm 2,6$ \\
\hline Tirosina & $72,4 \pm 4,1$ & $70,0 \pm 2,2$ & $71,9 \pm 7,0$ & $75,9 \pm 6,7$ \\
\hline Treonina & $72,6 \pm 9,8$ & $72,7 \pm 4,0$ & $70,8 \pm 5,2$ & $79,3 \pm 6,2$ \\
\hline Valina & $72,6 \pm 4,8$ & $76,2 \pm 8,4$ & $73,2 \pm 4,3$ & $78,7 \pm 9,6$ \\
\hline Aminas & Gorgonzola & Mussarela & Parmesão & Prato \\
\hline Agmatina & $70,1 \pm 2,2$ & $83,2 \pm 4,1$ & $71,1 \pm 2,8$ & $89,8 \pm 8,9$ \\
\hline Cadaverina & $60,5 \pm 2,4$ & $71,4 \pm 1,0$ & $71,2 \pm 2,2$ & $91,4 \pm 11,1$ \\
\hline Espermidina & $59,8 \pm 2,6$ & $71,0 \pm 0,8$ & $68,1 \pm 2,0$ & $84,8 \pm 11,2$ \\
\hline Espermina & $54,7 \pm 0,8$ & $79,2 \pm 1,7$ & $77,3 \pm 1,5$ & $81,2 \pm 11,6$ \\
\hline Feniletilamina & $68,6 \pm 6,0$ & $72,6 \pm 4,9$ & $64,7 \pm 3,7$ & $64,2 \pm 3,0$ \\
\hline Histamina & $70,7 \pm 4,1$ & $83,5 \pm 2,9$ & $75,7 \pm 3,9$ & $100,7 \pm 9,5$ \\
\hline Putrescina & $54,9 \pm 2,7$ & $71,2 \pm 2,9$ & $70,4 \pm 1,9$ & $79,7 \pm 11,0$ \\
\hline Serotonina & $103,1 \pm 3,6$ & $109,1 \pm 1,8$ & $99,0 \pm 5,5$ & $107,5 \pm 4,2$ \\
\hline Tiramina & $74,0 \pm 5,4$ & $85,6 \pm 3,9$ & $75,9 \pm 2,8$ & $89,8 \pm 5,3$ \\
\hline Triptamina & $84,9 \pm 2,9$ & $73,0 \pm 8,5$ & $98,9 \pm 2,2$ & $74,0 \pm 7,1$ \\
\hline
\end{tabular}


amostra sob condições definidas (BRASIL, 2011). Dessa forma o experimento foi realizado em seis (6) repetições com os extratos de cada tipo de queijo fortificados com o pool de aminoácidos e aminas bioativas em 3 níveis de concentração $(20,30$ e 40 pmol in column para aminoácidos e amônio, e 26, 40 e 53 pmol in column para aminas), sem variação de analista e dia de análise (cada queijo teve suas análises realizadas no mesmo dia com as mesmas soluções). O Manual de Garantia da Qualidade Analítica (BRASIL, 2011) preconiza coeficiente de variação $(\mathrm{CV})$ de, no máximo, 7,3\% para a precisão do método considerando a faixa de fortificação aplica$\mathrm{da}$, o que foi atingido na grande maioria dos resultados (Tabela 3). Porém, conforme recomendado pela Diretiva 657/2002 (COMISSÃO EUROPEIA, 2002), todos os coeficientes de variação encontrados estão dentro do esperado e aceitável para concentrações de analitos superiores a $1 \mathrm{mg} / \mathrm{kg}$, que é de $10 \%$.

\section{Recuperação}

A recuperação de aminoácidos, aminas e do íon amônio foi realizada em seis repetições e três níveis de fortificação para cada queijo estudado, a partir da etapa de extração dos analitos nos queijos. Dentre as determinações realizadas, $21,4 \%$ obtiveram recuperação acima de $80 \%, 56,2 \%$ entre 70 e $80 \%$ e $22,3 \%$ menor que $70 \%$ (Tabela 4 ). O queijo que apresentou melhores percentuais de recuperação foi o Prato. O Manual de Garantia da Qualidade Analítica (BRASIL, 2011) e a Diretiva 657/2002 (COMISSÃO EUROPEIA, 2002) recomendam recuperação entre $80 \mathrm{e}$ $110 \%$ para concentrações acima de $0,1 \mathrm{mg} / \mathrm{kg}$. A derivação aumenta a quantidade de etapas analíticas, aumentando o risco de perdas e diminuição da recuperação (LOIZZO et al., 2013). Além disso, o experimento de recuperação, quando realizado por fortificação de um pool de analitos (e não de analitos isola- dos), possui a limitação de uma etapa extra de preparo da solução concentrada com todos os analitos a serem testados, apresentando dificuldade de diluição conjunta e erros acumulados na pesagem de todos os compostos.

\section{Limites de detecção (LD) e quantificação (LQ)}

O limite de detecção é a menor concentração de um composto que o método é capaz de distinguir de zero confiavelmente, enquanto o limite de quantificação é a menor concentração que o método é capaz de quantificar de forma aceitável (BERNARDES; SOUZA, 2011).

A determinação dos limites de detecção (LD) e quantificação (LQ) pode ser realizada de diversas formas, segundo vários manuais de validação disponíveis, sendo o método mais utilizado o da relação sinal-ruído para técnicas analíticas em geral. Apesar disso, técnicas cromatográficas podem apresentar erros quando essa abordagem de medição é utilizada, uma vez que as condições cromatográficas podem afetar o resultado final (picos maiores aumentam a relação sinalruído, condições de uso e tipo da coluna também causam alterações), além do fato de a curva analítica ser construída com a área e não somente com o sinal do detector. Dessa forma, recomenda-se o cálculo do LD e LQ com base nos parâmetros da curva analítica, que é estatisticamente mais confiável. Assim o LD pode ser expresso como $3,3 \sigma / \mathrm{S}$ e o LQ como $10 \sigma / \mathrm{S}$, onde $\sigma$ é a estimativa do desvio padrão da resposta, que pode ser a estimativa do desvio padrão do branco, da equação da linha de regressão ou do coeficiente linear da equação e S é a inclinação ou coeficiente angular da curva analítica (RIBANI et al., 2004).

Com exceção do íon amônio, no qual o ponto mínimo da curva ficou entre o LD e o LQ (Tabela 5), para todos os outros analitos 
Tabela 5 - Limites de detecção (LD) e quantificação (LQ) para aminoácidos, aminase íon amônio, em mg/100g, conforme método apresentado

\begin{tabular}{|c|c|c|}
\hline \multirow{2}{*}{ Analitos } & \multicolumn{2}{|c|}{ Valor $(\mathrm{mg} / 100 \mathrm{~g})$} \\
\hline & LD & LQ \\
\hline Íon Amônio & 0,24 & 0,74 \\
\hline Aminoácidos & LD & LQ \\
\hline Ácido Aspártico & 0,81 & 2,46 \\
\hline Ácido Glutâmico & 0,21 & 0,64 \\
\hline Alanina & 0,34 & 1,02 \\
\hline Arginina & 0,22 & 0,67 \\
\hline Asparagina & 0,17 & 0,52 \\
\hline Cistina & 0,23 & 0,69 \\
\hline Fenilalanina & 0,16 & 0,49 \\
\hline Glicina & 0,13 & 0,38 \\
\hline Glutamina & 0,07 & 0,22 \\
\hline Histidina & 0,20 & 0,59 \\
\hline Isoleucina & 0,24 & 0,72 \\
\hline Leucina & 0,24 & 0,72 \\
\hline Lisina & 0,38 & 1,14 \\
\hline Metionina & 0,18 & 0,54 \\
\hline Prolina & 0,21 & 0,63 \\
\hline Serina & 0,43 & 1,31 \\
\hline Tirosina & 0,15 & 0,45 \\
\hline Treonina & 0,27 & 0,81 \\
\hline Valina & 0,25 & 0,75 \\
\hline Aminas & LD & LQ \\
\hline Agmatina & 0,09 & 0,26 \\
\hline Cadaverina & 0,21 & 0,63 \\
\hline Espermidina & 0,38 & 1,15 \\
\hline Espermina & 0,62 & 1,88 \\
\hline Feniletilamina & 0,16 & 0,49 \\
\hline Histamina & 0,11 & 0,34 \\
\hline Putrescina & 0,15 & 0,45 \\
\hline Serotonina & 0,36 & 1,09 \\
\hline Tiramina & 0,18 & 0,54 \\
\hline Triptamina & 0,15 & 0,44 \\
\hline
\end{tabular}

o menor ponto da curva ficou acima do LQ, mostrando que para compostos com concentração variando entre 2 e 100 pmol in column, após todo o processamento analítico, o método fornecerá resultados confiáveis de quantificação.

\section{CONCLUSÕES}

Foram apresentados parâmetros de desempenho para um método de cromatografia líquida de ultra eficiência para determinação de 19 aminoácidos, 10 aminas bioativas e íon amônio em queijos Parmesão, Gorgonzola, Prato e Mussarela. Conforme os resultados apresentados, o método é adequado para determinação dessas substâncias nos tipos de queijos estudados. Foi observado efeito de matriz para todos os tipos de queijo, o que indica a necessidade de utilizar a matriz para elaboração da curva de calibração, e não em solvente. A recuperação dos compostos nos queijos, embora tenha apresentado valores superiores a $70 \%$ em mais de $76 \%$ das determinações, pode ser reavaliada em condições experimentais que, ainda que mantenham as condições reais de análise, diminuam os erros intrínsecos das medições.

\section{AGRADECIMENTOS}

Os autores agradecem à FAPEMIG por bolsas e à FAPEMIG e ao CNPq pelos apoios financeiros concedidos.

\section{REFERÊNCIAS}

ALVAREZ, M. A.; MORENO-ARRIBAS, M. V. The problem of biogenic amines in fermented foods and the use of potential biogenic amine-degrading microorganisms as a solution. Trends in Food Science and Technology, v. 39, n. 2, p. 146-155, 2014.

BAKKER, J.; LAW, B. A. Cheese flavour. 
In: PIGGOTT, J. R.; PATERSON, A. (Eds.). Understanding Natural Flavours. $1^{\text {a }}$ ed. [s.1.] Springer Science, 1994. p. 283-297.

BERNARDES, A. C. M.; SOUZA, S. V. C. DE. Análise comparativa do guia para validação de métodos analíticos propostos pela ANVISA (RE no 899 de 2003) com o documento orientado do INMETRO e o protocolo internacional harmonizado pela AOAC International, ISO e IUPAC. Revista Analytica, v. 51, p. 66-77, 2011.

BRASIL. Ministério da Agricultura, Pecuária e Abastecimento. Manual de Garantia da Qualidade Analítica. Brasília: MAPA/ACS. 227 p. 2011.

COMISSÃO EUROPEIA. Diretiva 2002/657/ CE. Decisão da comissão de 12 de agosto de 2002 que dá execução ao disposto na Diretiva 96/23/CE do Conselho relativamente ao desempenho de métodos analíticos e à interpretação de resultados. Jornal Oficial das Comunidades Europeias, n. 221, p. 8-36, 2002.

CUSTÓDIO, F. B.; TAVARES, É.; GLÓRIA, M. B. A. Extraction of bioactive amines from grated Parmesan cheese using acid, alkaline and organic solvents. Journal of Food Composition and Analysis, v. 20, n. 3-4, p. 280288, 2007.

DUTRA, E. R. P.; MUNCK, A. V. Apostila de Fabricação de Queijos. EPAMIG, Instituto de Laticínios Cândido Tostes, 2002, 40 p.

EFSA PANEL ON BIOLOGICAL HAZARDS (BIOHAZ). Scientific Opinion on risk based control of biogenic amine formation in fermented foods. EFSA Journal, v. 9, n. 10, p. 1-93, 2011.

FIECHTER, G.; SIVEC, G.; MAYER, H. K.
Application of UHPLC for the simultaneous analysis of free amino acids and biogenic amines in ripened acid-curd cheeses. Journal of Chromatography B: Analytical Technologies in the Biomedical and Life Sciences, v. 927, p. 191-200, 2013.

FOX, P. F.; WALLACE, J. M. Formation of flavor compounds in cheese. Advances in Applied Microbiology, v. 45, p. 17-85, 1997.

GLÓRIA, M. B. A. Bioactive amines. In: HUI, Y. H. (Ed.). Handbook of Food Science, Technology and Engineering. [s.1.] Taylor \& Francis, 2006. p. 13.1-13.38.

HE, Y. et al. Simultaneous determination of food-related biogenic amines and precursor amino acids using in situ derivatization ultrasound-assisted dispersive liquid-liquid microextraction by ultra-high-performance liquid chromatography tandem mass spectrometry. Journal of Agricultural and Food Chemistry, v. 64, n. 43, p. 8225-8234, 2016.

JIA, S. et al. Simultaneous determination of 23 amino acids and 7 biogenic amines in fermented food samples by liquid chromatography/ quadrupole time-of-flight mass spectrometry. Journal of Chromatography A, v. 1218, n. 51, p. 9174-9182, 2011.

KABELOVÁ, I. et al. Determination of free amino acids in cheeses from the Czech market. Czech Journal of Food Science, v. 27, n. 3, p. 143-150, 2009.

LINARES, D. M. et al. Factors influencing biogenic amines accumulation in dairy products. Frontiers in Microbiology, v. 3, n. 180, p. 1-10, 2012.

LOIZZO, M. R. et al. Technological aspects and analytical determination of biogenic 
amines in cheese. Trends in Food Science \& Technology, v. 30, n. 1, p. 38-55, mar. 2013.

MAZZUCCO, E. et al. High-performance liquid chromatography-ultraviolet detection method for the simultaneous determination of typical biogenic amines and precursor amino acids. applications in food chemistry. Journal of Agricultural and Food Chemistry, v. 58, n. 1, p. 127-134, 2010.

McSWEENEY, P. L. H. Biochemistry of cheese ripening. In: FUQUAY, J. W.; FOX, P. F.; McSWEENEY, P. L. H. (Eds.). Encyclopedia of Dairy Sciences. 2a ed. [s.1.] Elsevier Ltd, 2011. p. 667-674.

RIBANI, M. et al. Validação em métodos cromatográficos e eletroforéticos. Química Nova, v. 27, n. 5, p. 771-780, 2004.
ROIG-SAGUÉS, A. X.; MOLINA, A. P.; HERNÁNDEZ-HERRERO, M. M. Histamine and tyramine-forming microorganisms in Spanish traditional cheeses. European Food Research and Technology, v. 215, n. 2, p. 96-100, 2002.

TUBEROSO, C. I. G. et al. Determination of dansylated amino acids and biogenic amines in Cannonau and Vermentino wines by HPLCFLD. Food Chemistry, v. 175, p. 29-35, 2015.

VALE, S.; GLÓRIA, M. B. A. Biogenic amines in Brazilian cheeses. Food Chemistry, v. 63, n. 3, p. 343-348, 1998.

YVON, M.; RIJNEN, L. Cheese flavour formation by amino acid catabolism. International Dairy Journal, v. 11, n. 4-7, p. 185201, 2001. 